\title{
The Primary Radical of a Submodule
}

\author{
Lamis J. M. Abulebda \\ University College, Abu Dhabi Universityn, Abu Dhabi, UAE \\ Email: lamis_jomah@yahoo.com
}

Received April 5, 2012; revised April 29, 2012; accepted May 7, 2012

\begin{abstract}
In this paper we introduced a definition for the primary radical of a submodule with some of its basic properties. We also define the P-radical submodule and review some results about it. We find a method to characterize the primary radical of a finitely generated submodule of a free module.
\end{abstract}

Keywords: Primary Submodule; Prime Radical of a Submodule; Radical Submodule; Free Module; Noetherian Module; Finitely Generated Submodule

\section{Introduction}

The prime radical of a submodule $N$ of an $R$-module $M$, denoted by $\operatorname{rad}_{M}(N)$ is defined as the intersection of all prime submodules of $M$ which contain $N$, if there exists no prime submodule of $M$ containing $N$, we put $\operatorname{rad}_{M}(N)=M \quad[1]$.

We naturally seek a counterpart in the primary radical of a submodule of module.

Firstly we introduced a definition for the primary radical of a submodule with some of its basic properties. We also define the $P$-radical submodule and review some results about it.

Finally, we find a method to characterize the primary radical of a finitely generated submodule of a free module.

\section{Some Basic Properties of the Primary Radical}

In this section we introduce the concept of the primary radical and give some useful properties about it.

\subsection{Definition}

The primary radical of a submodule $N$ of an $R$-module $M$, denoted by $\operatorname{prad}_{M}(N)$ is defined as the intersection of all primary submodules of $M$ which contain $N$. If there exists no primary submodule of $M$ containing $N$, we put $\operatorname{prad}_{M}(N)=M$.

If $M=R$, since the primary submodules and the primary ideals are the same, so if $I$ is an ideal of $R$, $\operatorname{prad}_{R}(I)$ is the intersection of all primary ideals of $R$, which contain $I$. Now, we give useful properties of the primary radical of a submodule.

\subsection{Proposition}

Let $N$ and $L$ be submodules of an $R$-module $M$. Then
1) $N \subseteq \operatorname{prad}_{M}(N)$
2) $\operatorname{prad}_{M}(N \cap L) \subseteq \operatorname{prad}_{M}(N) \cap \operatorname{prad}_{M}(L)$
3) $\operatorname{prad}_{M}\left(\operatorname{prad}_{M}(N)\right)=\operatorname{prad}_{M}(N)$

Proof.

1) It is clear.

2) Let $H$ be primary submodule of $M$ containing $L$, since $N \cap L \subseteq L \subseteq H$ so $\operatorname{prad}_{M}(N \cap L) \subseteq H$. Thus $\operatorname{prad}_{M}(N \cap L) \subseteq \operatorname{prad}_{M}(L)$. By the same way $\operatorname{prad}_{M}(N \cap L) \subseteq \operatorname{prad}_{M}(N)$. It follows $\operatorname{prad}_{M}(N \cap L) \subseteq \operatorname{prad}_{M}(N) \cap \operatorname{prad}_{M}(L)$.

3) By 1) we have $\operatorname{prad}_{M}(N) \subseteq \operatorname{prad}_{M}\left(\operatorname{prad}_{M}(N)\right)$. Now $\operatorname{prad}_{M}(N)=\bigcap L$ where the intersection is over all primary submodules $L$ of $M$ with $L \supseteq N$.

$$
\begin{aligned}
& \operatorname{prad}_{M}\left(\operatorname{prad}_{M}(N)\right) \\
& =\operatorname{prad}_{M}(\bigcap L) \subseteq \bigcap \operatorname{prad}_{M}(L)=\bigcap L
\end{aligned}
$$

In the following two propositions, we give a condition under which the other inclusion of 2) holds, that is; $\operatorname{prad}_{M}(N \cap L)=\operatorname{prad}_{M}(N) \cap \operatorname{prad}_{M}(L)$ provided that every primary submodule of $M$ which contains $N \cap L$ is completely irreducible submodule. Where a submodule $K$ of an $R$-module $M$ is called Completely Irreducible if whenever $N \cap L \subseteq K$, then either $N \subseteq K$ or $L \subseteq K$ where $N$ and $L$ are submodules of $M$.

\subsection{Proposition}

Let $N$ and $L$ be submodules of an $R$-module $M$. If every primary submodule of $M$ which contains $N \cap L$ 
is completely irreducible submodule, then:

$$
\operatorname{prad}_{M}(N \cap L)=\operatorname{prad}_{M}(N) \cap \operatorname{prad}_{M}(L) .
$$

Proof. By proposition (2.2, (2))

$\operatorname{prad}_{M}(N \cap L) \subseteq \operatorname{prad}_{M}(N) \cap \operatorname{prad}_{M}(L)$

If $\operatorname{prad}_{M}(N \cap L)=M$, clearly

$\operatorname{prad}_{M}(N)=\operatorname{prad}_{M}(L)=M$. If $\operatorname{prad}_{M}(N \cap L) \neq M$, there exists a primary submodule $K$ of $M$ such that, $N \cap L \subseteq K$ by hypothesis either $N \subseteq K$ or $L \subseteq K$ so that either $\operatorname{prad}_{M}(N) \subseteq K$ or $\operatorname{prad}_{M}(L) \subseteq K$, because every primary submodule containing $N \cap L$, so either $\operatorname{prad}_{M}(N) \subseteq \operatorname{prad}_{M}(N \cap L)$ or

$\operatorname{prad}_{M}(L) \subseteq \operatorname{prad}_{M}(N \cap L)$ therefore

$\operatorname{prad}_{M}(N) \cap \operatorname{prad}_{M}(L) \subseteq \operatorname{prad}_{M}(N \cap L)$.

\subsection{Proposition}

Let $N$ and $L$ be submodules of an $R$-module $M$ such that $\sqrt{[N: M]}+\sqrt{[L: M]}=R$, then $\operatorname{prad}_{M}(N \cap L)=\operatorname{prad}_{M}(N) \cap \operatorname{prad}_{M}(L)$.

Proof. If $K$ is a primary submodule containing $N \cap L$, then $\sqrt{[N \cap L: M]} \subseteq \sqrt{[K: M]}$. So

$\sqrt{[N: M]} \cap \sqrt{[L: M]} \subseteq \sqrt{[N \cap L: M]} \subseteq \sqrt{[K: M]}$.

Since $\sqrt{[K: M]}$ is a prime ideal, either

$\sqrt{[L: M]} \subseteq \sqrt{[K: M]}$ or $\sqrt{[N: M]} \subseteq \sqrt{[K: M]}$. If

$\sqrt{[N: M]} \subseteq \sqrt{[K: M]}$, then $\sqrt{[L: M]} \not \subset \sqrt{[K: M]}$ for

otherwise $R=\sqrt{[N: M]}+\sqrt{[L: M]} \subseteq \sqrt{[K: M]}$ which

is a contradiction. Therefore $N \subseteq K$. Now, applying proposition (2.3), we can conclude that

$\operatorname{prad}_{M}(N \cap L)=\operatorname{prad}_{M}(N) \cap \operatorname{prad}_{M}(L)$. We conclude the same result if $\sqrt{[L: M]} \subseteq \sqrt{[K: M]}$.

Let $N$ be a proper submodule of an $R$-module $M$. Let $P$ be a prime ideal of $R$. For each positive integer $n$, we shall denote by $K\left(N, P^{n}\right)$ the following subset of

$$
K\left(N, P^{n}\right)=\left\{m \in M \mid c m \in N+P^{n} M \text { for some } c \notin P\right\}
$$

\subsection{Proposition}

Let $N$ be a submodules of an $R$-module $M$ and $P$ be a prime ideal of $R$. For each positive integer $n$ : $K\left(N, P^{n}\right)=M$ or $K\left(N, P^{n}\right)$ is a $P$-primary submodule of $M$.

Proof. Let $n$ be any positive integer, it is clear that $K\left(N, P^{n}\right)$ is a submodule of $M$. Assume

$K\left(N, P^{n}\right) \neq M$. To show $K\left(N, P^{n}\right)$ is $P$-primary,
$P^{n} M \subseteq K\left(N, P^{n}\right)$ that is $P^{n} \subseteq\left[K\left(N, P^{n}\right): M\right]$. Now, let $L$ be a submodule of $M$ properly containing $K\left(N, P^{n}\right)$, let $r \in\left[K\left(N, P^{n}\right): L\right], r L \subseteq K\left(N, P^{n}\right)$.

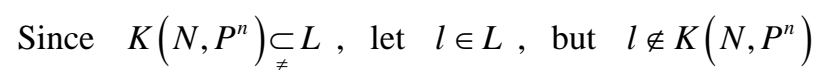
thus $r l \in K\left(N, P^{n}\right)$, there exists $c \notin P$ such that $c(r l) \in N+P^{n} M$. If $r \notin P$, then $c r \notin P$ and this implies $l \in K\left(N, P^{n}\right)$, which is a contradiction. It follows $r \in P$, therefore $\left[K\left(N, P^{n}\right): L\right] \subseteq P$.

So $K\left(N, P^{n}\right)$ is a primary submodule $P=\sqrt{\left[K\left(N, P^{n}\right): M\right]}$, we have proved above that $P^{n} \subseteq\left[K\left(N, P^{n}\right): M\right]$, that is $P \subseteq \sqrt{\left[K\left(N, P^{n}\right): M\right]}$.

Let $r \subseteq \sqrt{\left[K\left(N, P^{n}\right): M\right]}, r^{t} M \subseteq K\left(N, P^{n}\right)$ for some $t \in Z^{+}$, thus $c\left(r^{t} M\right) \in N+P^{n} M$ for some $c \notin P$. If $r \notin P$ then $c r^{t} \notin P$ this implies $M \subseteq K\left(N, P^{n}\right)$, which is a contradiction. Therefore $r \in P$ thus $\sqrt{\left[K\left(N, P^{n}\right): M\right]} \subseteq P$.

The following theorem gives a description of the primary radical of a submodule.

\subsection{Theorem}

Let $N$ be a submodule of a module $M$ over a Noetherian ring $R$. Then

$$
\begin{aligned}
& \operatorname{prad}_{M}(N) \\
& =\bigcap\left\{K\left(N, P^{n}\right) \mid P \text { is a primeideal of } R, n \in Z^{+}\right\}
\end{aligned}
$$

Proof. By proposition (2.2), for each positive integer $n$ and any prime ideal $P$ we have $K\left(N, P^{n}\right)$ is a $P$-primary submodule containing $N$. Hence

$$
\begin{aligned}
& \operatorname{prad}_{M}(N) \\
& \subseteq \cap\left\{K\left(N, P^{n}\right) \mid P \text { is a prime ideal of } R, n \in Z^{+}\right\}
\end{aligned}
$$

For every primary submodule $H$ containing $N$ with $P=\sqrt{[H: M]}$ there exists a positive integer $r$ such that $K\left(N, P^{r}\right) \subseteq H$. So

$$
\begin{aligned}
& \cap\left\{K\left(N, P^{n}\right) \mid P \text { is a prime ideal of } R, n \in Z^{+}\right\} \\
& \subseteq K\left(N, P^{r}\right) \subseteq H
\end{aligned}
$$

Thus

$$
\begin{aligned}
& \cap\left\{K\left(N, P^{n}\right) \mid P \text { is a prime ideal of } R, n \in Z^{+}\right\} \\
& \subseteq \operatorname{prad}_{M}(N) .
\end{aligned}
$$

We will give the following definition. 


\subsection{Definition}

A proper submodule $N$ of an $R$-module $M$ with $\operatorname{prad}_{M}(N)=N$ will be called P-Radical Submodule.

Now, we are ready to consider the relationships among the following three statements for any $R$-module $M$.

1) $M$ satisfies the ascending chain condition for pradical submodules.

2) Each p-radical submodule is an intersection of a finite number of primary submodules

3) Every p-radical submodule is the p-radical of a finitely generated submodule of it.

\subsection{Proposition}

Let $M$ be an $R$-module. If $M$ satisfies the ascending chain condition for p-radical submodule of $M$ is an intersection of a finite number of primary submoules.

Proof. Let $N$ be a p-radical submodule of $M$ and put $N=\bigcap_{i \in I} N_{i}$, where $N_{i}$ is a primary submodule for each $i \in I$, and the expression is reduced. Assume that $I$ is an infinite index set. Without loss of generality we may assume that $I$ is countable, then

$N=\bigcap_{i=1}^{\infty} N_{i} \subseteq \bigcap_{i=2}^{\infty} N_{i} \subseteq \bigcap_{i=3}^{\infty} N_{i} \subseteq \cdots$ is an ascending chain of p-radical submodules, since by proposition (2.2),

$$
\bigcap_{i} N_{i} \subseteq \operatorname{prad}_{M}\left(\bigcap_{i} N_{i}\right) \subseteq \bigcap_{i}\left(\operatorname{prad}_{M}\left(\bigcap_{i} N_{i}\right)\right)=\bigcap_{i} N_{i}
$$

By hypothesis this ascending chain must terminate, so there exists $j \in I$ such that $\bigcap_{i=j}^{\infty} N_{i}=\bigcap_{i=j+1}^{\infty} N_{i}$, whence $\bigcap_{i=j+1}^{\infty} N_{i} \subseteq N_{j}$ which contradicts that the expression $N=\bigcap_{i} N_{i}$ is a reduced. Therefore $I$ must be finite.

\subsection{Proposition}

Let $M$ be an $R$-module. If $M$ satisfies the ascending chain condition for p-radical submodules, then every p-radical submodule is the p-radical of finitely generated submodule of it.

Proof. Assume that there exists a p-radical submodule $N$ of $M$ which is not the p-radical of a finitely generated submodule of it. Let $m_{1} \in N$ and let $N_{1}=\operatorname{prad}_{M}\left(R m_{1}\right)$ so $N_{1} \subset N$, hence there exists $m_{2} \in N-N_{1}$. Let $N_{2}=\operatorname{prad}_{M}\left(R m_{1}+R m_{2}\right)$, then $N_{1} \subset N_{2} \subset N$, thus there exists $m_{3} \in N-N_{2}$, etc. This implies an ascending chain of p-radical submodules, $N_{1} \subset N_{2} \subset N_{3} \subset \cdots$ which does not terminate and this contradicts the hypothesis.

\subsection{Proposition}

Let $M$ be a finitely generated $R$-module. If every primary submodule of $M$ is the p-radical of a finitely generated submodule of it, then $M$ satisfies the ascending chain condition for primary submodules.

Proof. Let $N_{1} \subseteq N_{2} \subseteq N_{3} \subseteq \cdots$ be an ascending chain of primary submodules of $M$. Since $M$ is finitely generated then, $N=\bigcup_{i} N_{i}$ is a primary submodule of $M$.

Thus by hypothesis, $N$ is the p-radical for some finitely generated submodule $L=R\left(m_{1}, m_{2}, \cdots, m_{n}\right)$, hence $L \subseteq \operatorname{prad}_{M}(L)=N=\bigcup_{i} N_{i}$, then there exists $j \in I$ such that $L \subseteq N_{j}$ hence

$N=\operatorname{prad}_{M}(L) \subseteq \operatorname{prad}_{M}\left(N_{j}\right)=N_{j}$. Thus $\bigcup_{i} N_{i}=N_{j}$ for some $j \in I$. Therefore the chain of primary submodules $N_{i}$ terminates

\section{The Primary Radical of Submodules of Free Modules}

In this section we describe the elements of $\operatorname{prad}_{F}(N)$, where $N$ is a finitely generated submodule of the free module $F$. Let $n$ be a positive integer and let $F$ be the free $R$-module $R^{(n)}$.

Let $x_{i} \in F(1 \leq i \leq m)$ for some $m \in Z^{+}$, then $x_{i}=\left(x_{i 1}, x_{i 2}, \cdots, x_{i n}\right), \quad 1 \leq i \leq m$, for some $x_{i j} \in R$, $1 \leq i \leq m, 1 \leq j \leq n$.

We set

$$
\left[\begin{array}{llll}
x_{1} & x_{2} & \cdots & x_{m}
\end{array}\right]=\left(\begin{array}{cccc}
x_{11} & x_{12} & \cdots & x_{1 n} \\
x_{21} & x_{22} & \cdots & x_{2 n} \\
\vdots & \vdots & & \vdots \\
x_{m 1} & x_{m 2} & \cdots & x_{m n}
\end{array}\right) \in M_{m \times n}(R)
$$

Thus the $j$ th row of the matrix $\left[\begin{array}{llll}x_{1} & x_{2} & \cdots & x_{m}\end{array}\right]$ consists of the components of the element $x_{j}$ in $F$. Let $A=\left(a_{i j}\right) \in M_{m \times n}(R)$.

By a $t \times t$ minor of $A$ we mean the determinant of a $t \times t$ submatrix of $A$, that is a determinant of the form:

$$
\left|\begin{array}{ccc}
a_{i(1) j(1)} & \cdots & a_{i(1) j(t)} \\
\vdots & & \vdots \\
a_{i(t) j(1)} & \cdots & a_{i(t) j(t)}
\end{array}\right|
$$

where $1 \leq i(1) \leq \cdots \leq i(t) \leq m, \quad 1 \leq j(1) \leq \cdots \leq j(t) \leq n$. For each $1 \leq t \leq \min (m, n)$.

We denote by $A_{t}$ the ideal of $R$ generated by the $t \times t$ minors of $A$.

Note that $A_{1}=\sum_{i=1}^{n} \sum_{j=1}^{m} R a_{i j} \supseteq A_{2} \supseteq A_{3} \supseteq \cdots \supseteq A_{k}$, where $k=\min (m, n)$.

The key to the desired result is the following two propositions.

\subsection{Proposition}

Let $R$ be a ring and $F$ be the free $R$-module $R^{(n)}$, for some positive integer $n$. Let $N=\sum_{i=1}^{m} R x_{i}$ be a finitely generated submodule of $F$ where $m<n$. If 
$r \in \operatorname{prad}_{F}(N)$, then $\left[r x_{1} x_{2} \cdots x_{m}\right]_{t}$ in

$\bigcap\{P \mid$ for every maximal ideal $P$ such that

$$
\left.\left[x_{1} \cdots x_{n}\right]_{t} \subset P^{l} \text { for some } l \in Z^{+}\right\}, 1 \leq t \leq m+1
$$

Proof. Suppose $r=\left(r_{1}, r_{2}, \ldots, r_{n}\right) \in \operatorname{prad}_{F}(N)$ where $r_{i} \in R, 1 \leq i \leq n$. Let $P$ be any maximal ideal of $R$ and $\ell \in Z^{+}$such that $\left[0 x_{1} \cdots x_{m}\right]_{t} \subseteq P^{\ell}$. By proposition (2), there exists $c \in R \backslash P, \quad s_{i} \in R, \quad(1 \leq i \leq m)$ and $p_{i} \in P^{\ell}$ such that $c r=s_{1} x_{1}+s_{2} x_{2}+\cdots+s_{m} x_{m}+p$, where $p=\left(p_{1}, p_{2}, \cdots, p_{n}\right)$, that is, if $x_{i}=\left(x_{i 1}, x_{i 2}, \cdots, x_{i n}\right)$ where $x_{i j} \in R$ $(1 \leq i \leq m, 1 \leq j \leq n)$, then

3.1) $c r_{i}=s_{1} x_{1 i}+s_{2} x_{2 i}+\cdots+s_{m} x_{m i}+p_{i} ; 1 \leq i \leq n$

Suppose that

$1 \leq t \leq m+1$, let $1 \leq i(1) \leq i(2) \leq \cdots \leq i(t-1) \leq m$, $1 \leq j(1) \leq \cdots \leq j(t) \leq n$.

Let

$$
X_{t}=\left|\begin{array}{ccc}
r_{j(1)} & \cdots & r_{j(t)} \\
X_{i(1) j(1)} & \cdots & X_{i(1) j(t)} \\
\vdots & \ddots & \vdots \\
X_{i(t-1) j(1)} & \cdots & X_{i(t-1) j(t)}
\end{array}\right|
$$

which is a $t \times t$ minor of $\left[\begin{array}{lllll}r & x_{1} & x_{2} & \cdots & x_{m}\end{array}\right]$. Then by (3.1)

$$
\begin{aligned}
& c X_{t}=\left|\begin{array}{ccc}
c r_{j(1)} & \cdots & c r_{j(t)} \\
X_{i(1) j(1)} & \cdots & X_{i(1) j(t)} \\
\vdots & \ddots & \vdots \\
X_{i(t-1) j(1)} & \cdots & X_{i(t-1) j(t)}
\end{array}\right| \\
& =\sum_{h=1}^{m} S_{h}\left|\begin{array}{ccc}
x_{h j(1)} & \cdots & x_{h j(t)} \\
X_{i(1) j(1)} & \cdots & X_{i(1) j(t)} \\
X_{i(t-1) j(1)} & \cdots & X_{i(t-1) j(t)}
\end{array}\right| \\
& +\left|\begin{array}{ccc}
p_{j(1)} & \cdots & p_{j(t)} \\
X_{i(1) j(1)} & \cdots & x_{i(1) j(t)} \\
X_{i(t-1) j(1)} & \cdots & x_{i(t-1) j(t)}
\end{array}\right| \in P^{l} .
\end{aligned}
$$

which is primary with $c \notin P$ (note that, here $p_{j(1)}, \cdots, p_{j(t)} \in P^{\ell}$ ) hence $X_{t} \in P^{\ell} \subseteq P$ or $X_{t} \in P$. It follows $\left[\begin{array}{lllll}r & x_{1} & x_{2} & \cdots & x_{m}\end{array}\right]_{t} \in P$ for every maximal ideal $P$ with $\left[\begin{array}{lllll}0 & x_{1} & x_{2} & \cdots & x_{m}\end{array}\right]_{t} \in P^{\ell}$ for some $\ell \in Z^{+}$and $1 \leq t \leq m+1$

\subsection{Proposition}

Let $R$ be a ring and $F$ be the free $R$-module $R^{(n)}$, for some positive integer $n$. Let $N=\sum_{i=1}^{m} R x_{i}$ be a finitely generated submodule of $F$ where $m<n$. If $\left[\begin{array}{lllll}r & x_{1} & x_{2} & \cdots & x_{m}\end{array}\right]_{t}$ in
$\bigcap\left\{P^{l} \mid\right.$ for every prime ideal $P$ such that

$$
\left.\left[0 x_{1} \cdots x_{m}\right]_{t} \subset P^{l} \text { for some } l \in Z^{+}\right\}
$$

$1 \leq t \leq m+1$, then $r \in \operatorname{prad}_{F}(N)$.

Proof. Suppose

$\left[r x_{1} \cdots x_{m}\right]_{t} \in\left\{P^{l} \mid\right.$ for every prime ideal $P$

such that $\left[0 x_{1} \cdots x_{m}\right]_{t} \subset P^{l}$ for some $\left.l \in Z^{+}\right\}$

and $1 \leq t \leq m+1$. Let $P$ be any prime ideal of $R$ and $k$ any positive integer. It is enough to show that $r \in K\left(N, P^{k}\right)$ for all $\mathrm{k} \in \mathrm{Z}^{+}$.

If $\left[\begin{array}{lllll}0 & x_{1} & x_{2} & \cdots & x_{m}\end{array}\right]_{1} \in P^{k}$, then

$r_{i} \in\left[\begin{array}{lllll}r & x_{1} & x_{2} & \cdots & x_{m}\end{array}\right]_{1} \in P^{k}$, hence

$r=\left(r_{1}, r_{2}, \cdots, r_{n}\right) \in P^{k} F \subseteq K\left(N, P^{k}\right)$. Suppose SSS

$\left[\begin{array}{lllll}0 & x_{1} & x_{2} & \cdots & x_{m}\end{array}\right]_{1} \nsubseteq P$.

Note that $\left[\begin{array}{lllll}0 & x_{1} & x_{2} & \cdots & x_{m}\end{array}\right]_{m+1}=0 \in P^{k}$ for all $k$.

Thus there exists $1 \leq t \leq m$ such that

$\left[\begin{array}{lllll}0 & x_{1} & x_{2} & \cdots & x_{m}\end{array}\right]_{t} \nsubseteq P$, but $\left[\begin{array}{lllll}0 & x_{1} & x_{2} & \cdots & x_{m}\end{array}\right]_{t+1}$ is a subset of $P^{k}$, there exists

$1 \leq i(1) \leq \cdots \leq i(t) \leq m, 1 \leq j(1) \leq \cdots \leq j(t) \leq n$, such that

$$
d=\left|\begin{array}{ccc}
X_{i(1) j(1)} & \cdots & X_{i(1) j(t)} \\
X_{i(t) j(1)} & \cdots & X_{i(t) j(t)}
\end{array}\right| \notin P
$$

By hypothesis, for each $1 \leq j \leq n$

$$
\left|\begin{array}{cccc}
r_{j} & r_{j(1)} & \cdots & r_{j(t)} \\
x_{i(1) j} & x_{i(1) j(1)} & \cdots & x_{i(1) j(t)} \\
\vdots & \vdots & \ddots & \vdots \\
x_{i(t) j} & x_{i(t) j(1)} & \cdots & x_{i(t) j(t)}
\end{array}\right| \in P^{k}
$$

Expanding this determinant by first column we find that $d r_{j}+a_{i(1)} x_{i(1) j}+\cdots+a_{i(t)} x_{i(t) j} \in P^{k}$ where

$$
a_{i(h)}=(-1)^{h}\left|\begin{array}{ccc}
r_{j(1)} & \ldots & r_{j(t)} \\
x_{i(1) j(1)} & \cdots & x_{i(1) j(t)} \\
x_{i(h-1) j(1)} & \cdots & x_{i(h-1) j(t)} \\
x_{i(h+1) j(1)} & \cdots & x_{i(h+1) j(t)} \\
x_{i(t) j(1)} & \cdots & x_{i(t) j(t)}
\end{array}\right|
$$

For each $1 \leq h \leq t$.

Note that $d$ and $a_{i(h)}(1 \leq h \leq t)$ are independent of $j$. Thus

$$
d r_{j}+a i_{(1)} x i_{(1) j}+\cdots+a_{i(t)} x_{i(t) j} \rightarrow P^{k} \quad 1 \leq j \leq n .
$$

i.e. $d r \in R x_{1}+R x_{2}+\cdots+R x_{m}+P^{k} F=N+P^{k} F$ with $d \notin P$, hence $r \in K\left(N, P^{k}\right)^{m}$.Thus $r \in \operatorname{prad}_{F}(N)$.

\subsection{Proposition}

Let $M_{1}$ and $M_{2}$ be $R$-modules and 


$$
M=M_{1} \oplus M_{2}=\left\{\left(m_{1}, m_{2}\right) \mid m_{i} \in M_{i}, i=1,2\right\}
$$

Let $N$ be a proper submodule of $M_{1}$, then $x \in \operatorname{prad}_{M_{1}}(N)$ if and only if $(x, 0) \in \operatorname{prad}_{M}(N \oplus 0)$.

Proof: Suppose first that $x \in \operatorname{prad}_{M_{1}}(N)$. Let $K$ be any primary submodule of $M$ such that $N \oplus 0 \subseteq K$. Let $K^{\prime}=\left\{m \in M_{1} \mid(m, 0) \in K\right\}$. $K^{\prime}$ is a submodule of $M_{1}$ and if $K^{\prime} \neq M_{1}$ then $K^{\prime}$ is a primary submodule of $M_{1}$ since, if $r m \in K^{\prime}$ where $r \in R$ and $m \in M_{1}$, then $(r m, 0) \in K$, so $r(m, 0) \in K$, which is primary submodule of $M$, hence either $(m, 0) \in K$ thus $m \in K^{\prime}$ or $r^{n} M \subseteq K$ for some $n \in Z^{+}$that is, $r^{n}\left(M_{1} \oplus M_{2}\right) \subseteq K$, so $r^{n}\left(M_{1} \oplus 0\right) \subseteq r^{n}\left(M_{1} \oplus M_{2}\right) \subseteq K$, therefore $\left.\left(r^{n} M_{1} \oplus 0\right)\right)=r^{n}\left(M_{1} \oplus 0\right) \subseteq K$, thus $r^{n} M_{1} \subseteq K^{\prime}$ for some $n \in Z^{+}$, that is $r \in \sqrt{\left[K^{\prime}: M_{1}\right]}$. Hence $K^{\prime}$ is a primary submodule of $M_{1}$ containing $N$. Thus $x \in K^{\prime}$, so $(x, 0) \in K$. It follows $(x, 0) \in \operatorname{prad}_{M}(N \oplus 0)$. Conversely, suppose that $(x, 0) \in \operatorname{prad}_{M}(N \oplus 0)$. Let $Q$ be a primary submodule of $M_{1}$ such that $N \subseteq Q$. Then $Q \oplus M_{2}$ is a primary submodule of $M$ containing $N \oplus 0$. Hence $(x, 0) \in Q \oplus M_{2}$, that is $x \in Q$ so $x \in \operatorname{prad}_{M_{1}}(N)$.

Now, we have the main result of this section.

\subsection{Theorem}

Let $R$ be a ring and $F$ be the free $R$-module $R^{(n)}$, for some positive integer $n$. Let $N=\sum_{i=1}^{m} R x_{i}$ be a finitely generated submodule of $F$ where $m<n$. If $r \in \operatorname{prad}_{F}(N)$, then $\left[\begin{array}{lllll}r & x_{1} & x_{2} & \cdots & x_{m}\end{array}\right]_{t}$ in

$$
\bigcap\{P \mid \text { for every maximal ideal } P \text { such that }
$$

$$
\left.\left[0 x_{1} \cdots x_{n}\right]_{t} \subset P^{l} \text { for some } l \in Z^{+}\right\} \text {; }
$$

$1 \leq t \leq \min (m+1, n)$.

Proof. Let $k=\min (m+1, n)$. Suppose first $k=m+1$, that is $m<n$, by proposition (3.1), if $r \in \operatorname{prad}_{F}(N)$, then $\left[\begin{array}{lllll}r & x_{1} & x_{2} & \cdots & x_{m}\end{array}\right]_{t}$ in

$$
\begin{aligned}
& \bigcap\{P \mid \text { for every maximal ideal } P \text { such that } \\
& \left.\left[0 x_{1} \cdots x_{n}\right]_{t} \subset P^{l} \text { for some } l \in Z^{+}\right\} ;
\end{aligned}
$$

$1 \leq t \leq \min (m+1, n)$.

Now suppose $k=n$, i.e. $n \leq m+1$. Let $G=R^{(m+1)}$, $r=\left(r_{1}, r_{2}, \cdots, r_{n}\right), x i=\left(x_{i 1}, x_{i 2}, \cdots, x_{i n}\right)$ for some $r_{j} \in R$ and $x_{i j} \in R, \quad(1 \leq i \leq m, 1 \leq j \leq n)$. By proposition (3.3), $r \in \operatorname{prad}_{F}(N)$ if and only if $\left(r_{1}, r_{2}, \cdots, r_{n}, 0,0, \cdots, 0\right)$ in $\operatorname{prad}_{G}\left(N^{\prime}\right)$. Where

$$
N^{\prime}=\sum_{i=1}^{m} R\left(x_{i 1}, x_{i 2}, \cdots, x_{i n}, 0,0, \cdots, 0\right) .
$$

Now apply proposition (3.1) to obtain the result.

The following example will illustrate application of the proposition (3.2).

\subsection{Example}

Let $R=Z, F=Z^{3}$ and $N$ be the submodule $Z(1,3,5)+Z 2(1,1,1)$ of $F$. Then

$\left(r_{1}, r_{2}, r_{3}\right) \in \operatorname{prad}_{F}(N)$ if $3 r_{1}-r_{2}, 5 r_{1}-r_{3}, 5 r_{2}-r_{3}$ in $2 Z$ and $\left(r_{1}-2 r_{2}+r_{3}\right)=0$.

\section{REFERENCES}

[1] R. L. McCasland and M. E. Moore, "On Radicals of Submodules of Finitely Generated Modules,” Canadian Mathematical Bulletin, Vol. 29, 1986, pp. 37-39. 